Pastor-Prada, R., Vicente-Nicolás, G., López-Melgarejo, A.M. \& López-Núñez, N. (2022). Danza educativa en el aula actual: percepción y formación de los docentes en Primaria y Secundaria. Revista Electrónica Interuniversitaria de Formación del Profesorado, 25(1), 79-91.

DOI: https://doi.org/10.6018/reifop.473541

\title{
Danza educativa en el aula actual: percepción y formación de los docentes en Primaria y Secundaria
}

Raquel Pastor Prada, Gregorio Vicente Nicolás, Ana María López Melgarejo, Norberto López Núñez

Universidad de Murcia

\section{Resumen}

El objetivo principal de esta investigación ha sido analizar la percepción y la comprensión del concepto de Danza Educativa de docentes en activo de Educación Primaria y Secundaria en España. La muestra está formada por 198 profesoras y 107 profesores en ejercicio $(\mathrm{N}=309)$ de Educación Primaria y Secundaria. Para la recogida de información se diseñó un cuestionario ad hoc que contempla tres dimensiones: (1) datos descriptivos y de formación, (2) comprensión del concepto de Danza Educativa y autopercepción sobre la competencia docente para realizar actividades de movimiento y danza; (3) implementación, usos y beneficios de la danza en el aula. Los resultados revelaron que tres cuartas partes de los participantes recibieron ninguna o muy poca formación universitaria y complementaria sobre movimiento y danza, si bien la mitad de la muestra conocía bien la Danza Educativa y se sentía con la formación necesaria para implementarla en el aula. Los resultados visibilizaron diferencias significativas dependiendo de la edad de los participantes, la especialidad cursada y la etapa educativa en la que desarrollan su actividad docente.

\section{Palabras clave}

Danza educativa; formación del profesorado; educación musical; educación física. 


\title{
Dance Education in today's classroom: perceptions and training of Primary and Secondary teachers
}

\begin{abstract}
The main objective of this research was to analyse the perception and understanding of the concept of Dance Education of teachers of Primary and Secondary Education in Spain. The sample consisted of 198 male and 107 female teachers $(N=309)$ of Primary and Secondary School. For the collection of information, an ad hoc questionnaire was designed which includes three dimensions: (1) descriptive and training data, (2) understanding of the concept of Dance Education and self-perception of teaching competence to perform movement and dance activities; (3) implementation, uses and benefits of dance in the classroom. The results revealed that three quarters of the participants received no or very little university and further training in movement and dance, although half of the sample had a good understanding of the Dance Education and felt they had the necessary training to implement it in the classroom. The results showed significant differences depending on the age of the participants, the speciality studied and the educational stage in which they are teaching.
\end{abstract}

\section{Key words}

Dance education; teacher training; music education; physical education.

\section{Introducción}

El concepto de Danza Educativa o Educación a través de la Danza (Dance Education o Education through Dance) fue definido hace ya más de un siglo por el precursor de la Danza Moderna europea, Rudolf Laban, con la idea de devolver a la danza su importancia en el plano educativo y terapéutico (Baril, 1977). Para Laban (1975), el valor expresivo de la danza es prioritario a la belleza de la forma o la perfección técnica y aboga por la incorporación de la danza a la educación para preservar la espontaneidad innata del ser humano y fomentar su expresión creativa y artística. En EE.UU. otra pionera, Margaret H’Doubler, inicia el camino para la inclusión de la danza en el ámbito académico, implantando en 1926 la primera especialidad de Danza en el Departamento de Educación Física de la Universidad de Wisconsin, tras publicar en 1925 el libro Dance and its place in Education, en el que defiende las contribuciones de la danza a la educación moderna.

La Danza Educativa pertenece al ámbito de la educación estética y su principal finalidad es poner la danza al alcance de toda persona, para fomentar la expresión creativa a través del movimiento y beneficiar su desarrollo integral, corporal, emocional, intelectual y social (Haselbach, 1978; H'Doubler, 2002; Maschat, 2017). En este sentido, el concepto de Danza Educativa supone una nueva forma de entender las aplicaciones pedagógicas del movimiento y la danza, y la necesidad de propuestas metodológicas y didácticas específicas enfocadas al ámbito amateur, que puedan ser adaptadas a diferentes contextos. El alumno o alumna podrá entonces descubrir, experimentar, pensar por sí mismo o misma, y generar propuestas 
creativas de movimiento, enfatizando así el proceso creativo como base del aprendizaje (Pastor, 2017).

La Danza Educativa integrada dentro del ámbito de la educación artística y estética (Haselbach, 1978; McCutchen, 2006), comparte sus dos dimensiones principales: la estética, en base al desarrollo de la sensibilidad y el arte como un lenguaje expresivo y comunicativo para el desarrollo integral del individuo, y la social, que apuesta por el arte como vía para la comprensión de la diversidad cultural, la integración social y la transformación positiva y creativa de la realidad (Chalmers, 2003). La educación a través de la danza busca una vía para la reflexión crítica, personal y colectiva, la construcción de conocimiento, la transmisión de valores y la educación multicultural (Melchior, 2011).

Si atendemos a estas premisas, el concepto de Danza Educativa debería incluirse en los currículos escolares como materia capaz de enriquecer los lenguajes expresivos y artísticos, al tiempo que se integra fácilmente en otras áreas, siendo capaz de desarrollar múltiples modos de aprender. Si bien la danza está presente en los currículos españoles de las enseñanzas de régimen general desde la Ley Orgánica de Ordenación General del Sistema Educativo (LOGSE) de 1990, Ilama la atención la omisión del concepto de Danza Educativa si atendemos a "su datación (mediados del siglo XX), desarrollo y consolidación, tomando en cuenta que bajo esta disciplina transcurre la danza en los Sistemas Educativos Nacionales en geografías diversas, Reino Unido, Alemania, Estados Unidos, Argentina, Chile, entre otros" (Bustos, 2019, p. 176).

La danza en el currículo no aparece como una asignatura curricular propia, sino dentro de los contenidos disciplinares relacionados con el aprendizaje de la Música y la Educación Física tanto de Educación Primaria como Secundaria (Vicente, Ureña, Gómez \& Carrillo, 2010), no siendo esta decisión curricular exclusiva de España, sino común a la gran mayoría de países del mundo (Cañabate, Rodríguez \& Zagalaz, 2016). Además, su consideración es claramente inferior respecto a otras materias artísticas como Música, Plástica o Dramatización, lo que conlleva una escasa tradición investigadora y pedagógica en el campo del movimiento y la danza (Bustos, 2019; Lago \& Espejo, 2007). Se podría afirmar que la danza como materia coresponsable en la formación integral del estudiante aún no forma parte de la cultura escolar, a pesar de su inclusión curricular (currículum oficial) que no siempre lleva consigo el desarrollo de las enseñanzas preestablecidas en el aula (currículum real).

Desde el punto de vista de la formación, el movimiento y la danza poseen una escasa presencia en los distintos planes de estudios en la formación inicial de maestros de las universidades españolas (Bustos, 2019), así como en los estudios de posgrado habilitantes para la profesión en Educación Secundaria y Bachillerato (López \& Vicente, 2019). Estudios realizados en diversas regiones de nuestro país avalan que los especialistas de Educación Física conocen y valoran positivamente la danza y la expresión corporal como disciplinas, aunque son consideradas las menos relevantes dentro de los bloques de contenido de la asignatura y no las suelen incluir en sus prácticas docentes, aludiendo como uno de sus principales motivos la falta de cualificación y/o capacitación pedagógica para impartir estos contenidos curriculares tanto en la Educación Primaria, como en la Educación Secundaria (Archilla \& Pérez, 2012; Cañabate, et al., 2016; Conesa \& Angosto, 2017; Lago \& Espejo, 2007; Montávez, 2011; Robles, Abad, Castillo, Giménez, \& Robles, 2013; Troya \& Cuéllar, 2013). Robles et al. (2013) advierten cómo dentro de la etapa de Secundaria "es preocupante, que 
a pesar del alto porcentaje de docentes que afirman tener poca formación en el ámbito de la expresión corporal, muy pocos se interesan por continuar su formación en dicha parcela" (p. 174).

En relación con el profesorado de Música, en Educación Primaria una amplia mayoría de los docentes especialistas consideran el movimiento y la danza como contenido curricular de gran valor educativo, además de considerar la necesidad de poseer una formación suficiente para su adecuada implementación en el aula (Domínguez, 2012; Lago \& Espejo, 2007). Sánchez, Cañabate, Calbó \& Viscarro (2014) constatan una buena vinculación del trabajo corporal en actividades musicales, así como una presencia moderada de las actividades de movimiento y danza en la enseñanza musical de Educación Infantil y Primaria, si bien escasea el trabajo con la improvisación y la composición creativa de movimiento. En la Educación Secundaria, García-Carvajal, Marín-López, Ruiz-Ariza \& Martínez-López (2013) señalan que “el profesorado más joven, emplea como recurso la expresión corporal en la enseñanza musical, lo planifica y tiene mayor concepción sobre ella que el profesorado de mayor edad y con menos nivel de formación inicial" (p.315), además de revelar cómo este contenido influye en una mayor adquisición de las competencias de la asignatura.

Por último, es importante recordar que diversos autores coinciden en señalar la relación entre la escasa consideración de la danza como disciplina escolar y la exigua formación docente que recibe el profesorado en sus estudios iniciales en esta materia (Cañabate, et al., 2016; Conesa \& Angosto, 2017; García-Carvajal et al., 2013; Lago \& Espejo, 2007; López \& Vicente, 2019; Troya \& Cuéllar, 2013). Esta situación supone una incoherencia entre lo que ofertan los estudios universitarios y lo que solicitan los curricula educativos de Educación Primaria y Secundaria (Cunha, 2020; Troya \& Cuéllar, 2013).

A tenor de las ideas planteadas en esta sección introductoria, la finalidad u objetivo principal de la investigación es analizar la percepción y la comprensión del concepto de Danza Educativa de docentes en activo de Educación Primaria y Secundaria en España. Dicho objetivo se articula en los subsiguientes específicos: (1) conocer la formación inicial y continua relacionada con la Danza Educativa que recibe el profesorado en sus estudios, (2) conocer la compresión que poseen los docentes de la Danza Educativa y la autopercepción que tienen sobre su competencia profesional al respecto y (3) analizar la implementación y valoración de actividades de movimiento y danza en su práctica educativa.

\section{Metodología}

\section{Participantes}

de este estudio está conformada por 309 profesoras y profesores en ejercicio (198 mujeres y 107 hombres) de Educación Primaria y Secundaria. Dos tercios de los (216) pertenecían a la primera etapa educativa, 58 a Educación Secundaria y 28 impartían clase en ambas etapas. La especialidad más destacada ha sido la de Música (167), seguida por Educación Física (58), en tercer lugar, Generalista de Primaria (34) y el resto otras especialidades.

\section{Instrumento}

Para la recogida de información se diseñó un cuestionario ad hoc que contempla tres dimensiones: (1) datos descriptivos y de formación inicial, continua y complementaria sobre movimiento y danza, (2) conocimiento y comprensión del concepto de Danza Educativa, 
autopercepción sobre formación y competencia docente para realizar actividades de movimiento y danza; (3) prácticas educativas, implementación, usos y beneficios de la danza en el aula. La primera dimensión incluyó las variables sexo, edad, especialidad educativa y la etapa en la que se desarrolla la práctica docente, así como la formación recibida en el ámbito universitario y la formación continua cursada en relación con la danza para el ámbito escolar. La segunda dimensión comprendió dos variables: conocimiento de la terminología respecto al ámbito de la Danza Educativa y autopercepción del profesorado sobre sus competencias vinculadas a la realización de actividades de movimiento y danza. Por último, la tercera sección incorporó variables sobre la realización de actividades de danza en las aulas y razones que motivan dicha puesta en práctica.

El cuestionario fue sometido para validación mediante juicio de un Comité de Expertos formado por 6 profesores y profesoras universitarias de reconocido prestigio en el ámbito de la Didáctica de la Música y la Danza. Se utilizó una escala de validación Likert para el juicio de expertos en los parámetros de adecuación y pertinencia sobre los ítems que conforman las variables que obtuvo un valor medio de 4,58 sobre 5 y además se calculó el grado de fiabilidad mediante el Alfa de Cronbach obteniendo 0.897 sobre 1 , lo que demuestra que las variables del instrumento de recogida de datos tienen una buena fiabilidad. Por último, teniendo en cuenta los comentarios y observaciones de los expertos, se realizaron pequeñas modificaciones de los ítems en forma y contenido.

\section{Procedimiento}

Realizada la revisión de la literatura sobre el objeto de estudio se diseñó el instrumento de recogida de la información y posteriormente se inició su proceso de validación. Una vez finalizada la versión final del cuestionario, se pasó a formato digital a través de la herramienta Formularios Google y se generó un enlace web que se compartió a través de redes sociales, asociaciones y organismos relacionados con el profesorado de Música, de Educación Física y de Educación Primaria y Secundaria en general. El cuestionario incluía una carta de presentación que informaba sobre el objetivo del estudio y se garantizaba la confidencialidad de los datos obtenidos. Se estableció un plazo de 60 días para su cumplimentación.

\section{Análisis de datos}

Se realizó un análisis exploratorio inicial para la descripción de variables (frecuencias, medias y comprobación de supuestos paramétricos). Además, se llevó a cabo un análisis inferencial para estudiar las relaciones entre diferentes variables (Chi-cuadrado, correlación de Spearman y Alfa de (ronbach). Para la interpretación de resultados se aceptó un nivel de confianza del $95 \%$ y valores $r_{s}>.05$ para las correlaciones. La información fue analizada con el programa SPSS versión 24.

\section{Resultados}

A continuación, se presentan los resultados obtenidos estructurados según los objetivos del estudio.

a) Conocer la formación inicial y continua relacionada con la Danza Educativa que recibe el profesorado en sus estudios 
En relación con la formación sobre movimiento y danza recibida en la etapa universitaria, un $71 \%$ de los participantes afirmaron tener nada o poca formación, frente a un $29 \%$ que tuvo bastante o mucha. Se observó una relación significativa entre esta variable y la edad, de forma que a mayor edad de los participantes, menor había sido su formación en la universidad (Tabla 1). También se percibió una relación con la especialidad cursada y la etapa educativa en la que ejercían, siendo el profesorado de Música y de Educación Primaria el que mostraba mayor formación universitaria en movimiento y danza. No se observó ninguna relación entre este tipo de formación y el género.

Con respecto a la formación complementaria sobre movimiento y danza, un tercio del profesorado (36.7\%) no había recibido este tipo de formación y la mitad de los participantes afirmó tener poca formación. Por el contrario, un $11.6 \%$ indicó que tenía bastante y $4,7 \%$ mucha o muchísima. En referencia al tipo de formación adquirida, un 52\% había sido a través de cursos en entidades y organismos públicos, $20.2 \%$ mediante cursos en el ámbito privado y un 4.7\% había sido en estudios de máster. Se observaron relaciones entre esta formación y el género, la edad, la especialidad y la etapa educativa (Tabla 1), aunque no se constataron correlaciones entre dichas variables. También se constató una relación entre la formación en la etapa universitaria y la formación complementaria, $X^{2}(9)=26.521, p<.009$, pero igualmente con una correlación baja.

\section{Tabla 1}

Relación entre la formación y la práctica del movimiento/danza y las variables de género, edad, especialidad y etapa educativa

\begin{tabular}{|c|c|c|c|c|}
\hline & Género & Edad & Especialidad & $\begin{array}{c}\text { Etapa } \\
\text { Educativa }\end{array}$ \\
\hline \multicolumn{5}{|l|}{ F. Etapa Universitaria } \\
\hline Valor & .758 & 23.602 & 45.596 & 59.592 \\
\hline gl & 3 & 9 & 12 & 6 \\
\hline$p$ & .860 & .005 & .000 & .000 \\
\hline \multicolumn{5}{|l|}{ F. Complementaria } \\
\hline Valor & 18.805 & 47.159 & 39.876 & 23.422 \\
\hline gl & 4 & 12 & 16 & 8 \\
\hline$p$ & .005 & .000 & .001 & .003 \\
\hline \multicolumn{5}{|l|}{ Percepción Formación } \\
\hline Valor & 6.458 & 7.654 & 59.921 & 6.026 \\
\hline gl & 3 & 9 & 12 & 6 \\
\hline$p$ & .091 & .569 & .000 & .420 \\
\hline Concepto & & & & \\
\hline \multicolumn{5}{|l|}{ Educativa } \\
\hline Valor & 5.915 & 12.328 & 55.235 & 3.271 \\
\hline gl & 3 & 9 & 12 & 6 \\
\hline$p$ & .116 & .195 & .000 & .774 \\
\hline \multicolumn{5}{|l|}{ Realización Sesiones } \\
\hline Valor & 12.753 & 10.096 & 59.827 & 15385 \\
\hline gl & 5 & 9 & 12 & 6 \\
\hline$p$ & .005 & .343 & .000 & .017 \\
\hline
\end{tabular}


b) Conocer la compresión que poseen los docentes de la Danza Educativa y la autopercepción que tienen sobre su competencia profesional al respecto

Sobre la comprensión del concepto de Danza Educativa, el 56.5\% de los participantes conocía nada o poco este término, frente a un $44.5 \%$ que lo dominaba bastante o mucho. No se apreció ningún tipo de relación entre esta variable y el género, la edad o la etapa educativa del profesorado, pero sí con la especialidad, que indicó que los profesionales de Música conocían más este concepto que el resto de especialistas (Tabla 1). Igualmente, también se constató una relación entre esta variable y la formación en la etapa universitaria, $X^{2}(9)=57.541, p<$ .000, y la formación complementaria, $X^{2}(12)=68.843, p<.000$, y la percepción de tener la formación necesaria, $X^{2}(9)=211.056, p<.000$. En este último caso hubo una correlación positiva, $\left(r_{s}=.626, p<.000\right)$, cuanto mayor era el conocimiento sobre el concepto de Danza Educativa, mayor era la percepción sobre su competencia para implementar las actividades de movimiento y danza.

En relación con la percepción que tiene el profesorado sobre si tiene la formación necesaria para realizar actividades de movimiento y danza en el ámbito escolar, el número de participantes (53.1\%) que consideró que tenía nada o poca formación necesaria fue similar al que tenía bastante o mucha (46.9\%). No se observó ningún tipo de relación entre esta variable y el género, la edad o la etapa educativa del profesorado, pero sí con la especialidad, pues los docentes de Música se sentían más preparados que el resto de especialidades (Tabla 1). Asimismo, también estaban relacionadas la percepción de la capacidad para realizar este tipo de actividades y la formación en la etapa universitaria, $X^{2}(9)=56.852, p<.000$, y la formación complementaria, $X^{2}(12)=107.941, p<.000$. En ambos casos, cuanto mayor era la formación, más competentes se sentían para llevar a cabo actividades de movimiento y danza en el aula.

c) Analizar la implementación y valoración de actividades de movimiento y danza en la práctica educativa

En relación con la realización de sesiones de movimiento y danza en el aula, la mitad del profesorado afirmó llevar a la práctica ninguna (12.8\%) o pocas $(35.8 \%)$ sesiones, mientras que el resto hacía bastantes o muchas. Se apreciaron relaciones entre esta variable y el género, pues las mujeres efectuaban más sesiones que los hombres (Tabla 1). Asimismo, la especialidad y la etapa educativa también estaban relacionadas con las sesiones de movimiento y danza, siendo los docentes de Música y los de Educación Primaria quienes más realizaban. Se observó también una relación entre la implementación de estas sesiones y la formación en la universidad, $X^{2}(9)=40.442, p<.000$, la formación complementaria, $X^{2}(12)=$ 42.534, $p<.000$, la percepción de formación suficiente, $X^{2}(9)=100.179, p<.000$, y el conocimiento del concepto de Danza Educativa, $X^{2}(9)=79.227, p<.000$. No se constataron correlaciones entre estas variables, si bien aquellas referentes al concepto de Danza Educativa y la percepción de la formación necesaria alcanzaron valores cercanos a una correlación moderada.

Cuando se preguntó a los participantes para qué utilizaban el movimiento y la danza en sus aulas, más del $80 \%$ del profesorado manifestó que realizaban este tipo de actividades para contribuir al desarrollo integral del alumnado (Tabla 2). Casi dos tercios de la muestra también consideraron como motivo la mejora de las habilidades transversales y la participación en festivales escolares. La mitad del profesorado esgrimió como argumento para la realización del movimiento y la danza la utilización de las mismas como actividad de relajación y vuelta a 
la calma, así como para contribuir al desarrollo de las Inteligencias Múltiples. La razón menos seleccionada fue la que hacía referencia al cumplimiento de la programación.

\section{Tabla 2}

Motivos por los que el profesorado incluye movimiento y danza en las aulas

\begin{tabular}{lcc}
\hline \multicolumn{1}{c}{ Motivos } & $\mathrm{F}$ & $\%$ \\
\hline Para contribuir al desarrollo integral del alumnado & 266 & 82.9 \\
Para mejorar habilidades transversales: memoria, concentración, & 194 & 60,4 \\
disciplina... & 186 & 57.9 \\
Para las festividades escolares (Fin de curso, Navidad...) & 159 & 49.5 \\
Como actividad de relajación y vuelta a la calma & 158 & 49.2 \\
Para contribuir al desarrollo de las Inteligencias Múltiples & 139 & 43.3 \\
\hline Para cumplir con la programación & &
\end{tabular}

$(\mathrm{N}=321)$

Por último, cuando se relacionan estos motivos con las variables de formación y la práctica docente, en la abla 3 se puede observar que todas estaban relacionadas con la contribución al desarrollo integral del alumnado y las Inteligencias Múltiples (en este último caso exceptuando la Formación Universitaria). Se podría afirmar que dichos argumentos son más frecuentes cuanto mayor es la formación complementaria, la percepción sobre si tiene la formación necesaria para realizar estas actividades en el ámbito escolar, el conocimiento del concepto de Danza Educativa y la realización de sesiones de movimiento y danza. Igualmente, se constató una relación entre estas tres últimas variables y los participantes que seleccionaron como motivo la mejora de las habilidades transversales. También es interesante destacar que, salvo una excepción, no se constató ninguna relación entre la formación, así como de la práctica del movimiento/danza, y los argumentos de cumplimiento de la programación, la participación en festividades y la realización de actividades de relajación.

\section{Tabla 3}

Relación entre la formación y la práctica del movimiento/danza y los motivos para su realización

\begin{tabular}{lcccccc}
\hline & $\begin{array}{c}\text { Desarrollo } \\
\text { Integral }\end{array}$ & $\begin{array}{c}\text { Habilidades } \\
\text { Transversales }\end{array}$ & $\begin{array}{c}\text { Festividades } \\
\text { Escolares }\end{array}$ & $\begin{array}{c}\text { Relajación y } \\
\text { vuelta calma }\end{array}$ & $\begin{array}{c}\text { Inteligencias } \\
\text { Múltiples }\end{array}$ & $\begin{array}{c}\text { Cumplimiento } \\
\text { programación }\end{array}$ \\
\hline F. Etapa & & & & & &
\end{tabular}

Universitaria

Valor

$\begin{array}{rcccccc}\text { Valor } & 8.553 & 2.999 & 9.274 & 2.493 & 5.899 & 7.595 \\ \mathrm{gl} & 3 & 3 & 3 & 3 & 3 & 3 \\ p & .036 & .392 & .026 & .477 & .117 & .055 \\ \text { F. Complementaria } & & & & & & \\ \text { Valor } & 26.822 & 7.046 & 3.209 & 8.089 & 14.773 & 3.614 \\ \mathrm{gl} & 4 & 4 & 4 & 4 & 4 & 4\end{array}$




\begin{tabular}{lrcccccc} 
& $p$ & .000 & .133 & .523 & .088 & .005 & .461 \\
Percepción & & & & & & & \\
Formación & & & & & & & \\
& Valor & 43.223 & 18.315 & 1.340 & 5.123 & 32.322 & 4.044 \\
& $\mathrm{gl}$ & 3 & 3 & 3 & 3 & 3 & 3 \\
Concepto & $p$ & .000 & .000 & .720 & .163 & .000 & .257 \\
Edu. & Danza & & & & & & \\
& Valor & 39.849 & 12.002 & 2.722 & 4.512 & 8.616 & .617 \\
& gl & 3 & 3 & 3 & 3 & 3 & 3 \\
Realización & $p$ & .000 & .007 & .436 & .211 & .035 & .893 \\
Sesiones & & & & & & & \\
& Valor & 64.557 & 31.023 & 3.958 & 5.284 & 25.704 & 7.028 \\
& gl & 3 & 3 & 3 & 3 & 3 & 3 \\
& $p$ & .000 & .000 & .266 & .152 & .000 & .071 \\
\hline
\end{tabular}

\section{Discusión y conclusiones}

En relación con el primer objetivo del presente estudio, conocer la formación inicial y continua relacionada con la Danza Educativa que recibe el profesorado en sus estudios, los resultados obtenidos muestran que un $71 \%$ de los participantes afirmaron haber recibido nada o poca formación sobre movimiento y danza en su etapa inicial, frente a un $29 \%$ que tuvo bastante o mucha. Estos datos se corresponden con los obtenidos por García-Carvajal et al. (2013), quienes muestran que tan solo un 31\% de los docentes de Música de Educación Secundaria cursaron alguna asignatura relativa a la expresión corporal en sus estudios iniciales. Asimismo, si atendemos al contexto de la Educación Física, Troya \& Cuéllar (2013), en su estudio sobre la práctica educativa de la Danza, determinaron que un $24 \%$ de los docentes manifiesta no haber cursado asignaturas de ámbito expresivo en su formación inicial. Igualmente, Archilla \& Pérez (2012) afirman que para la mayoría de los profesores de Educación Secundaria los contenidos de expresión corporal recibidos en la formación universitaria resultaron insuficientes, con poca carga lectiva y de poca utilidad para su futuro desempeño docente.

Los resultados visibilizan una relación significativa entre la variable formación universitaria y la edad, de forma que, a mayor edad de los participantes, menor había sido su formación en la universidad, así como con especialidad cursada y etapa educativa en la que ejercen los docentes, siendo el profesorado de Música y de Educación Primaria el que muestra mayor formación universitaria en movimiento y danza. Estos datos coinciden con los recogidos por Lago \& Espejo (2007) quienes señalan diferencias porcentuales significativas entre los maestros de Educación Musical (76\%) y Educación Física (90\%), respecto a la autopercepción sobre la preparación recibida en movimiento y danza dentro de su formación inicial.

En relación con la formación complementaria relacionada con cursos de movimiento y danza, los porcentajes anteriores aumentan hasta un $83.7 \%$ de docentes que afirman habían recibido nada o poca, siendo el porcentaje que manifiesta no haber realizado ningún curso (36.7\%) sensiblemente menor al que recogen (62\%) otras investigaciones (Montávez, 2011; Troya \& Cuéllar, 2013). Parece que, tal y como afirman autores como Archilla \& Pérez (2012) y Robles 
et al. (2013), pese a ser contenidos sobre los que los docentes manifiestan haber recibido poca formación inicial, no muestran excesivo interés por mejorar su capacitación a través de la formación continua.

Con respecto al segundo objetivo del trabajo, conocer la compresión que poseen los docentes de la Danza Educativa y la autopercepción que tienen sobre su competencia profesional al respecto, el 56.5\% de los participantes conocía nada o poco el concepto de Danza Educativa, frente a un $44.5 \%$ que lo dominaba bastante o mucho, siendo variables determinantes para ello la especialidad de Música, la formación inicial y continua, así como la autopercepción sobre su competencia docente para implementar contenidos relacionados con el movimiento y la danza. Estos resultados resultan llamativos, dadas las diferencias porcentuales respecto a los resultados obtenidos en formación inicial y complementaria. En referencia con la autopercepción sobre la competencia profesional, aproximadamente la mitad de los participantes (53.1\%) consideró que tenía nada o poca formación para realizar actividades de movimiento y danza en el ámbito escolar, frente a la otra mitad que tenía bastante o mucha (46.9\%). Estos resultados contradicen los recogidos en otros estudios, como los de Lago \& Espejo (2007) quienes determinan que un $82 \%$ de los docentes no se consideraron preparados, ni siquiera parcialmente, en el área del movimiento y la danza, o Archilla \& Pérez (2012) para quienes la mayoría de los docentes de Educación Secundaria afirmaron haber recibido una insuficiente formación inicial, por lo que se sienten inseguros y carentes de recursos didácticos. Al igual que ocurría con la comprensión del concepto de Danza Educativa, los docentes de Música se sienten más preparados que el resto de especialidades, así como aquellos que tuvieron niveles más elevados de formación universitaria y complementaria.

En relación con el tercer objetivo planteado, analizar la implementación y valoración de actividades de movimiento y danza en la práctica educativa, se puede afirmar que la mitad del profesorado (49.4\%) aseguró llevar a la práctica ninguna o pocas sesiones de movimiento y danza en el aula. Estos datos coinciden con los resultados obtenidos por otros autores dentro del ámbito de la Educación Física (Archilla \& Pérez, 2012; Conesa \& Angosto, 2017; Montávez, 2011; Robles et al., 2013) que recogen una menor dedicación docente a contenidos relacionados con la danza y la expresión corporal, llegando a ser incluso inexistentes. También se ha apreciado una relación entre esta variable y el género, siendo las mujeres las que incluyen en mayor medida la expresión corporal y la danza en sus sesiones, resultado que coincide con otros autores (Conesa \& Angosto, 2017; García-Carvajal et al., 2013; Robles et al., 2013). Asimismo, se ha apreciado una relación significativa entre esta variable y la especialidad y etapa educativa, siendo los docentes de Música y de Educación Primaria quienes más implementan estas actividades.

También relacionado con el tercer objetivo y para indagar sobre la valoración de la Danza Educativa por parte del profesorado, se les preguntó sobre los motivos y usos a los que dedican el movimiento y la danza en sus prácticas docentes, manifestando más del $80 \%$ que realizaban este tipo de actividades para contribuir al desarrollo integral del alumnado, siendo la opción menos seleccionada para cumplir con la programación. En relación con las variables de formación e implementación docente, cabe destacar que cuanto mayor era la formación complementaria, la autopercepción sobre su capacitación docente, la comprensión del concepto de Danza Educativa, así como la implementación de contenidos afines, los argumentos más frecuentes fueron para contribuir al desarrollo integral del alumnado y al 
desarrollo de las Inteligencias Múltiples. Resulta interesante verificar que aquellos motivos que se vinculan con una menor valoración y comprensión de los principios de la Danza Educativa, el cumplimiento de la programación, la participación en festividades y la realización de actividades de relajación, no se relacionan, salvo una excepción, con la formación en la materia. Es decir, cuando los profesores tienen un mayor conocimiento del concepto de Danza Educativa, menos motivaciones "secundarias" argumentan para la práctica de contenidos de movimiento y danza. También se ha constatado que los docentes de Música argumentan la praxis de este arte en sus aulas con una mayor fundamentación y convicción respecto al desarrollo integral del alumnado.

Por último y a la luz de los resultados obtenidos, convenimos con Bustos (2019) en reclamar la consideración de la Danza Educativa como materia que beneficia la formación didáctica de los futuros docentes, dadas las relaciones positivas halladas entre el conocimiento del concepto de Danza Educativa, la autopercepción de los docentes respecto a su competencia profesional, la formación inicial y continua y la implementación en la práctica de aula de contenidos relacionados con el movimiento y la danza. Podemos concluir que cuanto mayor es el conocimiento que los profesores tienen sobre el concepto de Danza Educativa, mayor es la percepción sobre su capacitación docente para implementar actividades afines. La Danza Educativa puede ayudar a los futuros docentes no solo a experimentar el lenguaje de la danza, sino a conocer su didáctica específica para el aula y a dotarles de recursos metodológicos, estrategias de intervención y materiales didácticos para su adecuada implementación. Paralelamente, se atenderá a las necesidades que manifiestan los propios docentes al mismo tiempo que se reducirán las distancias entre la formación inicial que reciben y las exigencias de los curricula de Educación Primaria y Secundaria en relación con esta materia.

\section{Referencias}

Archilla, M. T. \& Pérez, D. (2012). Dificultades del profesorado de E.F. con las actividades de expresión corporal en secundaria. EmásF, Revista Digital de Educación Física, (3)14, 176190.

Baril, J. (1987). La Danza Moderna. Barcelona: Paidós.

Bustos, A. (2019). Evolución histórica de la danza en la universidad española (1983-2017) [tesis doctoral, Universidad de Salamanca]. Repositorio Institucional https://gredos.usal.es/handle/10366/140373.

Cañabate, D., Rodríguez, D. \& Zalagaz, M.L. (2016). Educación física y danza: valoración de su integración en las escuelas de primaria de las comarcas de Girona. Apunts. Educación Física y Deportes, 125, 53-62.

Chalmers, F. G. (2003). Arte, educación y diversidad cultural. Barcelona: Paidós.

Conesa, E. \& Angosto, S. (2017). La expresión corporal y danza en la educación física de secundaria y bachillerato. Cuadernos de Psicología del Deporte, (17)2, 111-120.

Cunha, D. S. S. (2020) A Integração das Artes na Formação Docente para a Educação Básica no Brasil e na Espanha [tesis de doctorado, Universidade Estadual Paulista]. Repositorio Institucional UNESP. http://hdl.handle.net/11449/192599. 
Domínguez, M. (2012). Opinión de los docentes de música sobre la praxis del movimiento y la danza en Educación Primaria. En P. Miralles \& A. B. Mirete (Eds.), La formación del profesorado en Educación Infantil y Educación Primaria. (pp. 143-149). Murcia: Servicio de publicaciones de la Universidad de Murcia.

García-Carvajal, E., Marín-López, J., Ruiz-Ariza, A. \& Martínez-López, E.J. (2013). La expresión corporal como medio de aprendizaje en la asignatura de música. Opinión del profesorado. Journal of Sport and Health Research, (5)3, 305-318.

Haselbach, B. (1978). Dance Education. London: Schott.

H'Doubler, M. N. (2002). Education through dance. En A. Mertz (Ed.), The Body can Speak. Essays on Creative Movement Education with Emphasis on Dance and Drama (pp.10-14). Southern Illinois University Press. (1st ed. 1957)

Laban, Rudolf (1975). Danza educativa moderna. Barcelona: Paidós. (1ª ed. 1948)

Lago, P. \& Espejo, A. (2007). El movimiento y la danza: su importancia dentro del curriculum de primaria. Educación y futuro: revista de investigación aplicada y experiencias educativas, 17, 149-164.

López, M. \& Vicente, G. (2019). Movimiento y danza en Educación Secundaria. ¿Un fraude curricular o un currículo fraudulento? Revista Interuniversitaria de Formación del Profesorado, 94(33.3), 213-234.

Maschat, V. (2017). La Danza al alcance de todos. En M. Rodríguez (Coord.), 1er Encuentro Salud en Movimiento (pp. 47-57). Madrid: Librería Deportiva Esteban Sanz.

McCutchen, B. P. (2006). Teaching Dance as Art in Education. Champaign, IL: Human Kinetics

Melchior, E. (2011) Culturally responsive dance pedagogy in the primary classroom, Research in Dance Education, (12)2, 119-135

Montávez, M. (2011). La Expresión Corporal en la realidad educativa. Descripción y análisis de su enseñanza como punto de referencia para la mejora de la calidad docente en los centros públicos de Educación Primaria de la ciudad de Córdoba [tesis doctoral, Universidad de Córdoba]. Repositorio Institucional http://hdl.handle.net/10396/6310

Pastor, R. (2017). Danza Educativa/Creativa: Hacia una pedagogía de la danza en la diversidad, la inclusión y la equidad. En B. Martínez del Fresno (Coord.), Danza, investigación y educación: Género e inclusión social (pp. 85-98). Málaga: Libargo.

Robles, J., Abad, M. T., Castillo, E., Giménez, F. J., \& Robles, A. (2013). Factores que condicionan la presencia de la expresión corporal en la enseñanza secundaria según el profesorado de educación física. Retos. Nuevas tendencias en Educación Física, Deporte y Recreación, 24, 171-175.

Sánchez, S., Cañabate, D., Calbó, M., \& Viscarro, I. (2014). Música, movimiento y danza: un enfoque integrador para la formación inicial del profesorado. Educatio Siglo XXI, 32(3), 145-158.

Troya, Y. \& Cuéllar, M. J. (2013). Formación docente y tratamiento de la danza en Canarias: evaluación desde la Educación Física. Retos. Nuevas tendencias en Educación Física, Deporte y Recreación, 24, 165-170. 
Vicente, G., Ureña, N., Gómez, M. y Carrillo, J. (2010). La danza en el ámbito educativo. Retos. Nuevas Tendencias en Educación Física, Deporte y Recreación, 17, 42-45. 